\title{
REVISTAACIÓN
}

Revista Educación

ISSN: 0379-7082

ISSN: 2215-2644

revedu@gmail.com

Universidad de Costa Rica

Costa Rica

\section{La crisis de la educación musical como consecuencia de la decadencia de la institución educativa}

\author{
-Alvarado, Rolando Angel \\ La crisis de la educación musical como consecuencia de la decadencia de la institución educativa \\ Revista Educación, vol. 42, núm. 2, 2018 \\ Universidad de Costa Rica, Costa Rica \\ Disponible en: http://www.redalyc.org/articulo.oa?id=44055139024 \\ DOI: https://doi.org/10.15517/revedu.v42i2.29055
}

Esta obra está bajo una Licencia Creative Commons Atribución-NoComercial-SinDerivar 3.0 Internacional. 
La crisis de la educación musical como consecuencia de la decadencia de la institución educativa

The Music Education Crisis as a Consequence of Educational Institution Decay

Rolando Angel-Alvarado [1]

Universidad Pública de Navarra, España

rolando.angel.alvarado@gmail.com

iD http://orcid.org/0000-0002-1800-2667

\author{
DOI: https://doi.org/10.15517/revedu.v42i2.29055 \\ Redalyc: http://www.redalyc.org/articulo.oa?id=44055139024
}

Recepción: 23 Mayo 2017

Aprobación: 20 Marzo 2018

\title{
Resumen:
}

La institución educativa está en crisis porque diversos elementos ponen en duda principios fundamentales como la equidad y la calidad. Este estudio revisa la cadena de valores que existe en la institución educativa, que abarca desde las agencias supranacionales hasta la escuela, desde la perspectiva de la educación musical. El método se enmarca en el diseño cualitativo, según la teoría fundamentada centrada en el método comparativo constante. Los resultados muestran que la decadencia de la institución educativa se replica en la educación musical y, lo más preocupante, las desigualdades socioeducativas surgen durante el diseño curricular. En conclusión, el enfoque sociomusical hace posible la superación de la crisis, ya que reconoce la complejidad del mundo cultural al promover una interacción sistemática entre la institución educativa y el resto de los grupos sociales en el mundo.

Palabras ClaVE: Sistema educativo, establecimientos de enseñanza, educación musical, política y planificación de la cultura, educación comparada.

\section{Abstract:}

The educational institution suffers a crisis due to diverse elements are casting of doubt essential principles of equity and quality. This study aims to review the values' string in educational institution, which encompasses from the Supranational agencies to school, according to music education perspective. The method is framed to qualitative design, according to grounded theory, which is centred on the Constant Comparative Method. The results show that educational institution decay is replicated on music education and, the most serious, the socio-educational inequality arises during curriculum design. In conclusion, sociomusical approach makes possible the crisis overcoming, since it recognises complexity of the cultural world by promoting a systematic interaction between the educational institution and the rest of social groups in the world.

KEYWORDS: Educational systems, educational institutions, music education, cultural policy and planning, comparative education.

\section{INTRODUCCIÓN}

Las agencias supranacionales tienen como factor común su interés por los principios fundamentales referidos a la equidad y la calidad de la educación. Todas reconocen a los gobiernos nacionales como los responsables por configurar y regular el sistema de escolarización. Igualmente, todas aspiran a que la educación proporcione las competencias necesarias para la vida activa y laboral de la sociedad, porque permite así dinamizar la economía y, por ende, el mercado laboral. Con todo esto, se puede observar un enfoque pragmático en el sistema educativo, fundado en el pragmatismo neoliberal (Schipper, 2014), lo cual se

\section{NotAS DE AUTOR}

[1] Doctorando en Humanidades y Ciencias Sociales de la Universidad Pública de Navarra (España), con financiamiento de la Comisión Nacional de Investigación Científica y Tecnológica de Chile [CONICYT]. Entre sus antecedentes destaca su beca doctoral CONICYT, su premio como investigador emergente otorgado por la European Educational Research Association el año 2016 y su labor académica en el Departamento de Educación de la Universidad de Chile entre los años 2013-2015. Sus líneas de investigación se vinculan a la gestión de sistemas educativos y la educación musical para la primera infancia, con publicaciones en diversos espacios académicos internacionales. 
reafirma al constatar que los Estados priorizan los intereses financieros por sobre las materias sociales durante las crisis económicas (Davies, 2014).

La equidad y la calidad son considerablemente complejas de abordar, no obstante, es necesario reflexionar periódicamente sobre la igualdad de oportunidades que ofrece la institucionalidad educativa para la formación musical estudiantil. Ciertamente, el constructo de institución educativa también es muy complejo, porque comprende desde las determinaciones de las agencias supranacionales, pasando por las regulaciones ministeriales y administrativas, hasta la implementación en la escuela y concreción en el aula de clases. Así, este análisis comprende la institución educativa como la cadena de valores que solo representa a la educación formal (Coll, 2013), lo que implica entender la educación musical como una asignatura escolar.

Considerando estos elementos, se puede aseverar que la educación musical está inmersa en un sistema curricular regulado por los intereses tecnocráticos y economicistas del pragmatismo neoliberal, factor general que explica por qué la educación musical ha perdido importancia en los planes de estudios promulgados por los niveles ministeriales (Aróstegui, 2016). Con el fin de determinar categorías conceptuales que permitan explicar con mayor especificidad la crisis de la educación musical en el sistema educativo obligatorio, se formulan las siguientes preguntas de investigación:

I. ¿Cómo se explica la crisis de la educación musical en el sistema educativo?

II. ¿Qué estrategias debe implementar la institución educativa para superar la crisis de la educación musical?

\section{MÉTodo}

Se utiliza la teoría fundamentada centrada en el método comparativo constante [MCC] porque la recogida y el análisis de datos es un proceso espiral que culmina con la saturación teórica de las categorías conceptuales (Carrero, Soriano y Trinidad, 2012). Así, los datos se recogen desde la bibliografía científica internacional, donde las etapas de comparación e integración de las categorías conceptuales (a través de su codificación abierta) son los procesos que hacen factible la delimitación teórica y la escritura de una teoría (San Martín, 2014).

En este estudio, el MCC responde al proceso deductivo porque busca conceptualizar y ampliar las categorías conceptuales para generar teoría sobre la crisis de la educación musical en el sistema educativo, sin necesariamente verificar la universalidad teórica o la incidencia de otras causas o propiedades (Osses, Sánchez y Ibáñez, 2006).

Así, se predeterminan las siguientes categorías conceptuales:

- La incidencia de la música comercial en la cultura escolar, tanto en el marco curricular como didáctico.

- La infraestructura escolar, entendida como el equipamiento objetivo que la escuela posee para impartir clases de educación musical.

- La formación pedagógica del profesorado que imparte la asignatura de música en las escuelas.

\section{OBJetivos}

Este estudio se plantea desde la pedagogía crítica, y establece los siguientes objetivos:

I. Analizar el rol de la institución educativa en el sistema social, desde la perspectiva de la educación musical.

II. Delimitar la teoría de la crisis de la educación musical. 
III. Proponer una teoría que incida en el círculo hermenéutico de la educación musical, que sirva como propuesta para la superación de la crisis de la asignatura.

\section{CONTEXTO SUPRANACIONAL}

La Carta de las Naciones Unidas (Organización de las Naciones Unidas [ONU], 1948), basándose en el respeto hacia el principio de igualdad de derechos, se compromete a promover la solución de problemas internacionales de carácter económico y social, junto con la cooperación internacional en el orden cultural y educativo. Así, ONU instituye que organismos especializados en las problemáticas mencionadas cuentan con amplias atribuciones intergubernamentales definidas en sus estatutos.

En este marco jurídico, la Organización de las Naciones Unidas para la Educación, la Ciencia y la Cultura [UNESCO, por sus siglas en inglés] surge como organismo especializado supranacional que se compromete con la visión holística y humanista de la educación, sosteniendo el principio que la educación es esencial para el desarrollo humano, social y económico (UNESCO, 2011).

La Declaración Universal de Derechos Humanos (Oficina Regional de Educación para América Latina y el Caribe de la UNESCO [OREALC/UNESCO], 2008), en su Artículo 26, señala que la educación debe ser gratuita y obligatoria durante la instrucción elemental y fundamental, la instrucción técnica y profesional debe ser generalizada y, el acceso a los estudios superiores debe asegurarse con criterios de equidad basados en los méritos académicos. Igualmente, asevera que la educación debe desarrollar la personalidad humana y el fortalecimiento del respeto a los derechos humanos, las libertades fundamentales y el mantenimiento de la paz, favoreciendo la amistad entre las naciones y todos los grupos étnicos o religiosos. Finalmente, indica que los padres y madres tienen el derecho preferente de escoger el tipo de educación que le brindarán a sus hijos e hijas.

UNESCO se compromete absolutamente con la Declaración Universal de Derechos Humanos, es más, reafirma el Derecho fundante que "toda persona tiene derecho a la educación" (OREALC/UNESCO, 2008, p. 12-13) al establecer el compromiso mundial de proporcionar educación básica de calidad a toda la población (UNESCO, 2014).

En el año 2010, UNESCO ha establecido durante la Agenda de Seúl los Objetivos para el Desarrollo de la Educación Artística. Estos velan que la educación artística sea (UNESCO, 2010): 1) accesible, esencial y sostenible en el tiempo; 2) de gran calidad educativa desde su concepción hasta su ejecución y; 3 ) una contribución para solucionar problemas sociales y culturales actuales. Así, UNESCO ampara la educación artística conforme con los criterios que establece la Declaración Universal de los Derechos Humanos, de modo que su acceso debe ser universal e inalienable.

El derecho a la educación debe ser garantizado por cada Estado que ratifique los instrumentos internacionales relativos a los derechos humanos (UNESCO, 2008), es crucial que los distintos sectores sociales establezcan compromisos estratégicos a largo plazo. Los gobiernos tienen la obligación de elaborar planes estratégicos para asegurar el derecho a la educación en los distintos niveles (UNESCO, 1990), sin embargo, las buenas intenciones políticas han quedado en el papel (UNESCO, 2008), porque no se están determinando auténticas decisiones en favor del mejoramiento de la equidad y la calidad de la educación. Es más, el panorama internacional se está complejizando porque hay una creciente participación de agentes privados (con fines de lucro o no) en materias educativas, los cuales, poco a poco, están irrumpiendo en las decisiones gubernamentales (UNESCO, 2015).

Por su parte, la Organización para la Cooperación y el Desarrollo Económicos [OCDE] establece que un sistema educativo implica equidad cuando se basa en la justicia y la inclusión (OCDE, 2012), mientras que la calidad es determinada por las competencias que estudiantes incorporan y desarrollan para su integración existosa al mercado laboral (OCDE, 2005). Por consiguiente, en los últimos años los países miembros han 
aumentado el gasto real por estudiante en todos los niveles educativos, han disminuido la tasa de desempleo y han aumentado la matrícula en educación terciaria (OCDE, 2016).

\section{Procedimientos}

El rol social de la institución educativa y la crisis de la educación musical en el sistema educativo son interpretados desde el proceso de codificación abierta, es decir, dicha información es recogida directamente desde la bibliografía científica para su análisis. Así, la codificación abierta permite ejecutar las primeras etapas del MCC, ya que garantiza la consistencia del proceso de identificación de las relaciones (comparación e integración) entre dichas categorías, dando lugar a una codificación axial que representa la interacción entre la institución educativa y la crisis de la educación musical, desde las categorías conceptuales predeterminadas (música comercial, infraestructura escolar y formación docente) que delimitan la teoría.

A partir de lo anterior, se lleva a cabo un proceso de codificación selectiva con el fin de obtener una categoría central que exprese la crisis de la educación en el sistema educativo, según las codificaciones abiertas y axiales que la investigación arroje. Ciertamente, la categoría central no necesariamente se asume como hipótesis, sino que también se puede expresar en forma narrativa durante la escritura de la teoría (San Martín, 2014). Adicionalmente, esta investigación no pretende únicamente hacer un diagnóstico sobre la crisis de la educación musical, sino que también se propone enunciar una teoría que se pueda implementar en un plan de mejora con la intención de avanzar hacia la superación de la crisis.

\section{ANÁLISIS DE RESULTADOS Y DISCUSIÓN}

\section{La institución educativa desde la perspectiva de la educación musical}

Según Drucker (1993), la familia, la comunidad y la sociedad se definen por un lazo sociocultural que une a los seres humanos, mientras que las organizaciones sociales responden a colectivos de especialistas que se aúnan por una tarea común. Por ejemplo, la escuela se concentra en los procesos pedagógicos y las orquestas se enfocan en ofrecer recitales y conciertos. Actualmente, existe una sociedad de organizaciones porque todos los bienes y servicios son comercializados por instituciones expertas que cuentan con las certificaciones que la norma exige, dando lugar a un capital cultural institucionalizado (Bourdieu, 2005) que no solo refleja una multiplicidad de paradigmas en el escenario social, sino que da cuenta de un bosque paradigmático (Barker, 2000) en una misma organización. De hecho, la institución establece normas que determinan los comportamientos sociales y laborales para tener éxito, pero al mismo tiempo, cada estamento interno define sus propios paradigmas, por ejemplo: Dirección escolar, profesorado, estudiantes, asistentes de la educación, padres y madres y personas apoderadas, etc.

En esta complejidad paradigmática, el sistema educativo debe cumplir tres funciones esenciales (Dubet y Martuccelli, 1998). Primeramente, la selección escolar asigna calificaciones que impactan en el mercado laboral y en la jerarquización social del estudiantado, por lo tanto, tiene utilidad social. En segundo lugar, la educación se entiende como el proyecto de producción de un sujeto que no se ajusta a su utilidad social del todo, ya que la escuela intenta transformarlo desde fuera de la sociedad, en un recinto amurallado. Por último, la socialización se comprende como la producción de un sujeto adaptado a la sociedad según la posición social que le corresponde, por lo tanto, los grupos de estudiantes han interiorizado normas y aptitudes que les serán de utilidad para desempeñar su rol en la sociedad.

Las funciones de selección, educación y socialización dan cuenta del tradicional esquema educativo para transformar los valores en normas y las normas en roles, con una tensión muy fuerte entre las funciones (Dubet, 2010). Así, el funcionamiento de la institución educativa es inestable, porque reproduce las 
relaciones sociales (virtudes y defectos) que existen en la sociedad. Por ejemplo, estudiantes que provienen de familias con desventajas socioeconómicas en los países miembros OCDE obtienen un bajo rendimiento en PISA y muestran mayores dificultades para finalizar la educación secundaria superior (OCDE, 2016). Esta segregación social impide aceptar la escuela como una institución garante de la igualdad de oportunidades y la justicia social.

Por lo tanto, la institucionalidad educativa es la que está en crisis, porque no hay valores centrales (Dubet, 2010) entre los distintos estamentos educativos que conforman la cadena de valor. Por un lado, OCDE y sus países miembros se comprometen con el enfoque eficientista que configura una relación vertical entre las instituciones, negando el diálogo y limitándose a "asistencializar" al estudiantado, ignorando sus variables ontológicas e históricas (Freire, 1985). Por otro lado, UNESCO (2015) enfatiza que el enfoque humanístico debe ser el fundamento y el propósito para la educación del siglo XXI, respaldando así al magisterio que implementa pedagogías que superan las históricas dicotomías entre los aspectos cognitivos, emocionales y éticos (Acton y Glasgow, 2015; Lemberger y Hutchison, 2014).

En la educación musical de España se observa la incapacidad de integración entre los distintos niveles de la institucionalidad. El Ministerio de Educación, Cultura y Deporte [MECD] a través de la Ley Orgánica para la Mejora de la Calidad Educativa [LOMCE] ha eliminado del currículo obligatorio en los niveles de educación primaria a la educación artística (conformada por educación plástica y educación musical), con la intención de mejorar los resultados en las pruebas internacionales y cumplir las recomendaciones y acuerdos que se estipulan en el marco de la Estrategia Europea 2020 (MECD, 2013). Así, la LOMCE ignora la Agenda de Seúl y, además, no considera la octava competencia clave recomendada por el Parlamento Europeo y el Consejo de la Unión Europea (2006): conciencia y expresión culturales.

En todo caso, conforme a las estipulaciones de la LOMCE, la decisión curricular finalmente queda en manos de cada una de las 17 comunidades autónomas que, conforme con sus recursos, pueden incluir la educación artística en el marco del currículo optativo, según la organización curricular que disponga. Es decir, pueden optar por impartir ambas disciplinas artísticas equitativamente, priorizar una por sobre la otra, excluir una de las disciplinas, o derechamente no incorporar la educación artística en el currículo escolar. Hasta ahora, la gran mayoría de las comunidades han establecido estrategias para impartir transversalmente la educación musical (Belletich, Wilhelmi y Angel-Alvarado, 2016), de modo que están intentando corregir las contradicciones artístico-culturales que existen entre la LOMCE y las competencias clave (Alsina, 2012).

Más allá de los esfuerzos que realizan la mayoría de las comunidades autónomas para impartir la educación musical en la educación primaria, la LOMCE y su principio de selectividad provoca una dispersión curricular (Belletich et al., 2016) que amenaza con gestar progresivamente una desigualdad sociomusical, porque de momento, no todo el estudiantado tiene igualdad de oportunidades para formarse musicalmente.

El panorama español no es un caso aislado, sino que se replica de distintas formas en otros países. Aróstegui (2016) señala que, en el mundo, la educación musical pierde fuerza en la escuela porque las reformas educativas defienden modelos curriculares centrados en el mejoramiento de los resultados académicos en pruebas estandarizadas, lo que repercute negativamente en los recursos que se asignan a la asignatura. Todo esto puede significar que la educación musical está en crisis en el sistema educativo, pero es necesario hacer una distinción, la educación musical experimenta una crisis interna que se gatilla por la decadencia de la institución educativa (Dubet, 2010). Esto no quiere decir que la institución educativa ya no funcione, sino que se hace referencia a la carencia de valores centrales, debido a la compleja diversidad pedagógica que existe en la estructura estratégica de la educación. De hecho, los conflictos en la educación musical se fundan principalmente en la confrontación valórica de los enfoques pedagógicos, como también por la diversidad de modelos didácticos (Jorquera, 2010). La decadencia de la institucionalidad educativa responde a los siguientes motivos (Dubet, 2011): 
- La escuela pierde el monopolio del conocimiento con la irrupción de las tecnologías de la información y comunicación [TIC], porque hoy en día cualquier persona puede recavar la información que necesita en internet.

- El modelo de realización personal para la actividad profesional representa al docente por sus competencias. Es decir, el profesorado de música es reconocido por sus conocimientos en la disciplina, sin esperarse un alto desempeño en otras áreas.

- La escuela democrática de masas acoge a todo el estudiantado con sus problemas bio-psico-sociales e intereses personales, de modo que se incrementa la influencia de la escuela sobre la sociedad. Por consiguiente, la institución educativa debe asumir diversos roles bio-psico-sociales (alimentación, salud, educación, etc.).

- Se instala el mercado educativo, donde toda la ciudadanía espera rendimientos óptimos y el mercado laboral demanda competencias de utilidad social, de modo que la institución educativa pierde autonomía al tener que rendirle cuentas a la sociedad. Además, se instalan diversos enfoques pedagógicos (religiosos, laicos, conductistas, constructivistas, holísticos, etc.) que resaltan la carencia de valores centrales.

- La figura estudiantil deja de ser un recipiente de conocimiento y pasa a ser un sujeto activo que se construye a sí mismo. Es decir, el estudiantado asume el protagonismo en la actividad educativa.

\section{La decadencia institucional en la crisis de la educación musical}

Los síntomas de decadencia de la institucionalidad educativa se observan en el marco de la educación musical, incluso pueden dar cuenta del conflicto de enfoques pedagógicos que hay en la asignatura. Por ejemplo, un estudio realizado en Nicaragua (Cruz y Intxausti, 2013) ha reportado que la población estudiantil de primaria consume mucha televisión y música comercial, pero muestra escaso interés por las bellas artes y lo más grave, no tiene interés en la música tradicional local. Este caso da cuenta de la irrupción de los medios de comunicación en el imaginario infantil, ya que a pesar del afán docente por inculcar en el estudiantado sus raíces culturales, el cuerpo estudiantil manifiesta que sus intereses personales se orientan hacia la música comercial.

La dependencia a la música comercial ha transformado a la sociedad en consumidora de productos musicales (Longueira, 2013), de modo que la educación musical se ve limitada en términos de repertorio, contenidos y didácticas. Es más, en algunas situaciones didácticas la figura docente carece de sentido porque su rol mediador entre el contenido musical y sus estudiantes se vuelve poco claro (Georgii-Hemmingy Westvall, 2010). Por ejemplo, un grupo de docentes estadounidenses le atribuye un alto valor pedagógico a la música comercial, pero reconoce que no se siente preparado para abordarla en una situación didáctica, porque sus experiencias responden al ámbito personal (Springer, 2015). Si alguna persona de este grupo de docentes estuviera familiarizado con el rock y eventualmente, un estudiante decidiera interpretar música electrónica, porque ya posee conocimientos y cuenta con el equipamiento: ¿tendría sentido la presencia del personal docente en la situación didáctica?

La educación musical en Finlandia también ha sufrido los embates de la música comercial, porque la asignatura históricamente se ha orientado hacia la música nacionalista y las bellas artes, pero la pluralidad musical de la actualidad ha gatillado la incorporación de la música comercial en el aula (Heimonen y Hebert, 2010). La asignatura es obligatoria desde la enseñanza primaria hasta el primer año de educación secundaria superior (estudiantes de trece años de edad), posteriormente es de carácter optativo en el currículo, lo cual preocupa porque las personas jóvenes no preferirían la asignatura por su carácter tradicionalista (Anttila, 2010). Con estos elementos y otros, se ha discutido en Finlandia si el profesorado de música pierde libertad pedagógica con la irrupción del mercado global y la música comercial, como también si estos nuevos 
“enemigos" producen un estudiantado acrítico, ya que se limita a consumir productos musicales (Heimonen y Hebert, 2010).

Esta orientación funcional hacia la música comercial da cuenta del enfoque eficientista que predomina en el sistema educativo, porque hace que la mayoría de los sujetos se formen como consumidores musicales y otros pocos, los de mayor "talento", desarrollen competencias técnicas para desenvolverse como músicos recreadores o creadores conforme a sus posiciones sociales. Así, la educación musical reproduce las realidades sociales que existen en la sociedad. Por un lado, estudiantes que pertenecen a las clases sociales menos favorecidas reciben educación musical en la escuela por medio de estilos musicales propios de su contexto social marginal (Söderman y Sernhede, 2016), lo cual hace preservar los roles sociales y, por ende, la segregación de clases (Beach y Sernhede, 2013). Por otro lado, los sujetos con mayor "talento musical" asumen un rol activo en la economía local ya que, por ejemplo, los conservatorios de música en Londres logran aportar a la ciudad cuantiosas ganancias económicas cada año a raíz de recitales, conciertos y otras actividades (London School of Economics and Political Science, 2012).

Por consiguiente, el sistema educativo garantiza que unas pocas personas se desarrollen musicalmente para la vida activa y laboral de acuerdo con su posición social, lo cual confirma las funciones de selección, educación y socialización en la educación musical. Es importante señalar que hasta ahora este análisis se ha restringido a materias epistémicas, pero en este punto es necesario incluir otras variables de carácter concreto.

La educación artística en Colombia se estructura curricularmente por cuatro núcleos artísticos (música, plástica, teatro y danza), con la intención de transformar la escuela democrática de masas en un espacio cultural comunitario; sin embargo, las escuelas públicas no cuentan con la infraestructura adecuada para impartir el área musical (Lorenzo-Quiles y Cardenas, 2010). La realidad colombiana no es distinta a lo que acontece en otros países latinoamericanos, ya que en términos infraestructurales, el aula de música es casi inexistente en las escuelas primarias de la mayoría de los países de América Latina (Duarte, Gargiulo y Moreno, 2011; Duarte, Juareguiberry y Racimo, 2017). Estos datos son concluyentes, por lo tanto, los principios de equidad y calidad en la educación musical de latinoamerica no se aseguran transversalmente (Angel-Alvarado y Lira-Cerda, 2017), lo que provoca una distribución injusta de los sujetos en el espacio social, producto de la inestable relación que existe entre las funciones de selección, educación y socialización.

En materias de recurso docente, la mayoría de los Ministerios de Educación proponen al educador generalista para que imparta la asignatura de educación musical en la etapa primaria (Vaillant, 2013), sin embargo, aquellos grupos profesionales reciben una formación pedagógica general que no necesariamente profundiza en didácticas específicas de la educación musical. Así, la tipología del país de origen del profesorado determina sus necesidades formativas en la música (Giráldez y Palacios, 2016), ya que en México se solicita formación musical, en Ecuador se demanda formación en educación musical, en Colombia se requiere una formación interdisciplinar de la música, mientras que en Argentina, Chile y España, se solicita formación para la gestión de proyectos musicales y en materias de la didáctica musical, lo que muestra baja demanda formativa relacionada con los contenidos musicales. Por lo tanto, la formación del profesorado generalista debiese asegurar la artículación entre los contenidos pedagógicos y musicales, de lo contrario, la práctica docente será producto del ensayo y error (Touriñan y Longueira, 2009).

En otras realidades occidentales acontecen problemas similares. Por ejemplo en Reino Unido, el profesorado generalista que imparte clases de música (de carácter obligatorio) en general carece de confianza para enseñar la asignatura y lo más preocupante, asume que las altas capacidades musicales estudiantiles responden a excepciones de la regla y no a procesos de desarrollo (Pitts, 2012). Este caso confirma que la poca profundización en didáctica de la música durante la formación del profesorado generalista es un problema occidental, por lo tanto, es necesario establecer planes de mejora (o innovación) ante la contingencia internacional que aboga por una educación musical de calidad.

La innovación educativa debe surgir desde la macro-institucionalidad conformada por los estamentos ministeriales y las agencias supranacionales, porque si bien algunos centros de formación docente están 
tomando medidas al otorgar menciones en Música (Argentina, Chile, España, Reino Unido, entre otros), la ausencia de valores centrales hace que estos esfuerzos tengan poco impacto en el sistema educativo. En esta línea, es conveniente observar el caso de Brasil, ya que a través de programas ministeriales ha sabido transitar desde la escasa formación musical del profesorado generalista (Figueiredo, 2004) hacia la formación especializada en música del mismo conglomerado (Figueiredo, 2010). Por consiguiente, la innovación no debe avanzar hacia la exclusión del profesorado generalista del sistema de educación musical, sino que debiese orientarse hacia su formación competencial pedagógico-musical, desde la regulación del Ministerio de Educación, que debe garantizar la determinación de los valores centrales de los que hasta ahora carece la institución educativa.

Es importante señalar que la formación didáctica del profesorado especialista de educación musical también es diversa, ya que algunas instituciones buscan formar músicos o músicas que posean conocimientos sobre educación, mientras que otras se centran en la preparación pedagógica basada en el dominio de competencias musicales (Aranda, Carrillo y Casals, 2017; Poblete, 2017). Este panorama también hace cuestionar la adquisición y desarrollo de las competencias didácticas durante los programas de formación docente. Por lo tanto, no es apropiado discutir sobre la ideoneidad del profesorado especialista para todos los niveles educativos, en desmedro del sector generalista, sino que es imperioso discutir sobre la escasa formación didáctica que recibe el profesorado en su conjunto, tanto en su formación inicial como continua.

Con toda esta delimitación teórica, se obtiene una categoría central que expresa que el fenómeno de la crisis de la educación musical es gatillada por los síntomas de decadencia de la institución educativa, porque desde la génesis del diseño curricular no se aseguran los principios fundamentales de equidad y calidad para la educación, por lo tanto, la educación musical vulnera dichos derechos fundamentales mucho antes de que la situación didáctica se realice concretamente en el aula de clases. Todo esto demuestra la carencia de valores centrales en el sistema educativo formal, ya que las tensiones valóricas se traducen en el sistema social como desigualdades asociadas a cuestiones demográficas, sociomusicales, socioeconómicas, tecnológicas, entre otras. En concreto, la decadencia de la institución educativa gatilla la crisis de la educación musical porque:

- La música comercial enfrenta una serie de obstáculos para su incorporación en los planes de estudio, lo cual deja en evidencia la poca flexibilidad que tiene la asignatura para adaptarse a la demanda social.

- La infraestructura escolar genera inequidad sociomusical, ya que muchas escuelas latinoamericanas ni siquiera cuentan con la implementación mínima necesaria para impartir educación musical de calidad. Esta situación provoca una tensión entre la Agenda de Seúl y las determinaciones educativas internas (nacionales y locales).

- La formación en educación musical del profesorado no articula los contenidos pedagógicos con los musicológicos, dando lugar a una multiplicidad de representaciones pedagógicas sobre la didáctica de la música en el contexto escolar.

Si se incorporan a la matriz de análisis otras variables escolares (masificación escolar, mercado de la educación, recursos condicionados al desempeño, etc.), probablemente se haría más visible la reproducción de las relaciones sociales y, por ende, las inequidades de clases.

\section{Observar fuera de la institucionalidad: Hacia la superación de la crisis}

La institución educativa debe ser capaz de adaptarse al contexto social en la que está inmersa, tomando en cuenta sus características estratégicas, demográficas y tecnológicas, como también sus grados de incertidumbre (Hatch, 2013). En otras palabras, la institución educativa debe derribar los muros 
institucionales que ha levantado históricamente para establecer lazos de cooperación con otras agrupaciones sociales de la comunidad (Berkovich, 2014).

Un primer grupo es la familia del estudiantado, porque su influencia cultural es clave para el desarrollo musical de cada escolar. Por un lado, la asociación entre la práctica musical escolar y la personalidad infantil es determinada principalmente por la disposición artística y musical de las figuras parentales, incluso cuando se controlan las variables demográficas, cognitivas y de personalidad (Corrigall y Schellenberg, 2015). Por otro lado, las familias negocian su identidad musical. Por ejemplo, los hábitos musicales de adolescentes influyen en las experiencias musicales de las madres (Morgan, MacDonald y Pitts, 2015), ya que ellas generalmente muestran una apertura afectiva para conocer los gustos musicales de sus hijos e hijas. Con estos antecedentes, es fundamental que la institucionalidad educativa tome consciencia de la significancia de la familia en el proceso educativo-musical, porque son claves para el desarrollo musical de estudiantes y son un banco de información para la determinación epistémica del currículo.

Otro grupo son los centros de educación informal, porque las habilidades e intereses entre la institución educativa formal y las organizaciones culturales son complementarias. Su presencia en el espacio social no se puede entender como una competencia, sino que deben aunar esfuerzos para trabajar cooperativamente con la intención de elaborar proyectos socioeducativos en conjunto (Vierger, 2014), que tengan impacto en la reducción de las brechas culturales entre estudiantes, el intercambio cultural, la generación de audiencias y el compromiso musical (Pitts, 2007). Asímismo, la institución educativa debe generar vinculos con agrupaciones sociales locales que no se dediquen al rubro de la educación, pero que sí tengan implicaciones bio-psico-sociales en el estudiantado (hospitales, orquestas, residencias de personas adultas mayores, etc.), porque dichas relaciones organizacionales fomentan el sentido de pertenencia del estudiantado con su comunidad, como también pueden contribuir en la gestión de proyectos socioeducativos.

Un último grupo son los medios de comunicación masiva, porque han irrumpido tanto en la rutina diaria del hogar, que se han transformado en la actividad a la que asignan mayor cantidad de tiempo los grupos escolares (Liceras, 2014). Ciertamente, es complejo establecer acuerdos con este conglomerado, porque existen diversas plataformas comunicacionales (radio, televisión, redes sociales, etc.); no obstante, la clave radica en establecer lazos con los medios informativos locales para generar vínculos con la comunidad. Por ejemplo, asistir en vísperas de fiestas a la radio local para que el coro escolar interprete canciones de su repertorio durante una transmisión. Indiscutiblemente, internet también representa un espacio de alta relevancia porque facilita el proceso de compartir material, al punto de que el personal docente puede sugerir audiciones a sus estudiantes sin estar limitado por el período lectivo al que corresponde su clase.

Si la institución educativa no es capaz de considerar estas agrupaciones sociales, difícilmente superará la crisis. De hecho, superar la crisis desde el interior de la estructura estratégica es poco viable, porque los planes de mejora siempre apuntan a la colectividad estudiantil; pero, en educación musical, la situación didáctica está centrada en la interacción entre la persona, la música y el contexto social, de modo que la innovación educativa se debe focalizar principalmente en la persona, porque todo ser humano posee un propio e irrepetible bagaje sociomusical, producto de sus más íntimas experiencias estéticas.

Con todo esto, la institución educativa debe asumir que su interrelación con otras agrupaciones sociales es imperiosa porque aporta valores centrales al proceso educativo, generando un sistema armónico clave para la construcción de la identidad sociomusical de sus estudiantes (Guerrero, 2007). Este enfoque sociomusical implica la superación de las históricas confrontaciones entre las agrupaciones sociales y la institución educativa, de modo que valora los conocimientos musicales adquiridos antes de ingresar al sistema educativo y los desarrollados fuera de la escuela, como también los conocimientos incorporados en el espacio escolar formal. Por lo tanto, el enfoque sociomusical no reduce la educación musical a un mero espacio de socialización y transmisión cultural, sino que la reconoce como un espacio de encuentro crítico, lúdico, musical y educativo (Georgii-Hemming y Westvall, 2010), donde interactúa la figura docente, el contenido musicológico, la figura estudiantil y el contexto social. 
Según el enfoque sociomusical, la identidad es comprendida desde el pensamiento complejo ya que considera las relaciones entre el ser humano, la sociedad, la vida y el mundo (Tobón, 2005). Desde la perspectiva técnica, el enfoque sociomusical aporta en el diseño de situaciones didácticas contextualizadas y en la construcción e implementación de innovaciones socioeducativas. Desde la perspectiva teórica, el enfoque sociomusical proporciona, por un lado, conocimiento sobre la dimensión personal, al indagar en la identidad sociomusical de la persona y, por el otro, información sobre la dimensión social al establecer grados de equidad (Schmidt, 2015) y democratización (Samper, 2016) que existen en un contexto concreto, mediante el principio de diferenciación denominado nivel sociomusical. Así, se postula el enfoque sociomusical como un modelo útil para el análisis de la educación musical y su complejidad social.

\section{Conclusiones}

La educación musical pierde importancia curricular en el sistema educativo porque las políticas nacionales se han ido centrando en el mejoramiento de los resultados académicos en pruebas estandarizadas, con la intención de formar una ciudadanía que se integre exitosamente al mercado laboral. Este complejo panorama transnacional muestra que la escuela tiene una utilidad social que no necesariamente se orienta hacia la reducción de las brechas sociales, especialmente porque los informes PISA indican que el sistema educativo formal reproduce la realidad social. Por lo tanto, la institución educativa actualmente está en decadencia, porque carece de valores centrales que aseguren los derechos fundamentales de equidad y calidad de la educación. Al analizar el rol de la institución educativa en el sistema social, desde la perspectiva de la educación musical, es posible observar cómo la postura eficientista de las reformas educativas provoca una crisis en la asignatura musical, ya que esta pierde progresivamente sus recursos económicos, temporales y materiales.

En respuesta a la primera pregunta de investigación, el fenómeno de la crisis interna de la educación musical se expresa a través de los síntomas de decadencia de la institución educativa que plantea Dubet, porque desde la génesis del diseño curricular no se aseguran los principios fundamentales de equidad y calidad de la educación, por lo tanto, la educación musical vulnera dichos derechos fundamentales mucho antes de que la situación didáctica irrumpa concretamente en el aula de clases, instancia donde recién interactúan estudiantes y docentes, la musicología y el contexto. Así, se puede delimitar la teoría de la crisis de la educación musical, la cual asevera que la carencia de valores centrales en la institución educativa genera tensiones valóricas que se traducen como desigualdades (sociomusicales, demográficas, etc.) en el sistema social.

Como la institución educativa no ha sido capaz de asegurar los principios fundamentales, es imperioso que comiencen a irrumpir en el diseño curricular otros grupos sociales, derribando así los históricos muros que han aislado la escuela de la comunidad. En respuesta a la segunda pregunta de investigación, el trabajo colaborativo multi-organizacional que implica el enfoque sociomusical puede ser útil para reducir las brechas sociomusicales, porque, desde una perspectiva bio-psico-social y ética, se asume la educación musical como un espacio de encuentro crítico, lúdico, musical y educativo. Específicamente, dicho enfoque sociomusical se propone como una teoría que incide en el círculo hermenéutico de la educación musical, ya que aportaría conocimiento micro y macrosocial, y permitiría indagar en la identidad sociomusical del individuo y del contexto social, favoreciendo grados de equidad y democracia con base en principios de diferenciación propios del capital sociomusical. Por todo esto, el enfoque propuesto sirviría para superar la crisis en la educación musical.

Para concluir, la crisis de la educación musical se gatilla por los síntomas de la decadencia de la institución educativa. Por lo tanto, el enfoque sociomusical se postula como una teoría que contribuiría a la superación de la crisis disciplinar. Desde la perspectiva técnica, el enfoque sociomusical aporta conocimiento práctico para la implementación de situaciones didácticas contextualizadas, mientras que desde la perspectiva teórica, el enfoque sociomusical proporciona conocimiento sobre la identidad sociomusical de la persona y la 
comunidad. Con todo esto, el enfoque sociomusical tiene utilidad social, porque puede implementarse en el ciclo hermeneutico que existe entre la teoría y la práctica empírica.

\section{Agradecimientos}

El autor agradece la beca doctoral otorgada por la Comisión Nacional de Investigación Científica y Tecnológica (CONICYT) de la República de Chile.

\section{REFERENCIAS}

Acton, R. y Glasgow, P. (2015). Teacher wellbeing in Neoliberal contexts: A review of the literature. Australian journal of teacher education, 40(8), 99-114. doi: https://doi.org/10.14221/ajte.2015v40n8.6

Alsina, P. (2012). La competencia cultural y artística es un puente entre la escuela y el mundo exterior. En P. Alsina y A. Giráldez (Eds.), 7 ideas clave: La competencia cultural y artística (pp. 15-35). Barcelona: Grao.

Angel-Alvarado, R. y Lira-Cerda, J. P. (2017). Instalaciones y recursos educativos para la educación musical según la representación social de los estudiantes chilenos. Revista Electrónica de LEEME, 20, 19-31. doi: https:// doi.org/10.7203/LEEME.40.10412

Anttila, M. (2010). Problems with school music in Finland. British journal of music education, 27(3), 241-253. doi: https://doi.org/10.1017/S0265051710000215

Aranda, R., Carrillo, C., \& Casals, A. (2017). Formación del profesorado de música en Chile: Dos casos en la ciudad de Valparaíso. Debates, 18, 248-278.

Aróstegui, J. L. (2016). Exploring the global decline of Music Education. Arts education policy review, 117(2), 96-103. doi: https://doi.org/10.1080/10632913.2015.1007406

Barker, J. (2000). Paradigmas: El negocio de descubrir el futuro. Bogotá: McGraw-Hill.

Beach, D. y Sernhede, O. (2013). On creativity and resistance in Nordic youth culture on the margins. En G. Gudmundsson; V. Vestel y D. Beach (Eds.), Young people and marginalisation: Young people from immigrant families in Scandinavia (pp. 61-84). Londres: Tufnell Press.

Belletich, O., Wilhelmi, M. R. y Angel-Alvarado, R. (2016). La educación musical en la formación básica en España: El problema de la dispersión curricular. Perspectiva educacional, 55(2), 158-170. doi:10.4151/07189729-Vol.55Iss.2-Art. 454

Berkovich, I. (2014). A socio-ecological framework of social justice leadership in education. Journal of educational administration, 52(3), 282-309. doi: https://doi.org/10.1108/JEA-12-2012-0131

Bourdieu, P. (2005). Capital cultural, escuela y espacio social. Buenos Aires: Siglo XXI.

Carrero, V., Soriano, R. y Trinidad, A. (2012). Teoría fundamentada Grounded theory: El desarrollo de la teoría desde la generalización conceptual. Madrid: Centro de Investigaciones Sociológicas.

Coll, C. (2013). La educación formal en la nueva ecología del aprendizaje: Tendencias, retos y agenda de investigación. En J. L. Rodríguez (Ed.), Aprendizaje y educación en la sociedad digital (pp. 156-170). Barcelona: Universitat de Barcelona. DOI:10.1344/106.000002060

Corrigall, K. y Schellenberg, E. (2015). Predicting who takes music lesson: parent and child characteristics. Frointiers in psychology, 6(282), 1-8. doi: https://doi.org/10.3389/fpsyg.2015.00282

Cruz, J. y Intxausti, N. (2013). La educación artística en Nicaragua: Una investigación en el marco de la cooperación educativa Iberoamericana. Revista iberoamericana de educación, 61, 143-158.

Davies, W. (2014). Neoliberalism: A Bibliographic Review. Theory, Culture \& Society, 0(0), 1-9. doi: https:// doi.org/10.1177/0263276414546383

Drucker, P. (1993). La sociedad postcapitalista. Barcelona: Apóstrofe. 
Duarte, J., Gargiulo, C. y Moreno, M. (2011). Infraestructura escolar y aprendizajes en la educación básica latinoamericana: Un análisis a partir del SERCE. Washington D.C.: Banco Interamericano de Desarrollo, División de Educación.

Duarte, J., Juareguiberry, F. y Racimo, M. (2017). Suficiencia, equidad y efectividad de la infraestructura escolar en América Latina según el TERCE. Santiago: Oficina Regional de Educación para América Latina y el Caribe (OREALC/UNESCO Santiago).

Dubet, F. (2010). Sociología de la experiencia. Madrid: Complutense.

Dubet, F. (2011). La experiencia sociológica. Barcelona: Gedisa.

Dubet, F. y Martuccelli, D. (1998). En la escuela: Sociología de la experiencia escolar. Buenos Aires: Losada.

Figueiredo, S. (2004). A preparaçâo musical de professores generalistas no Brasil. Asociación brasileña de educación musical, 12(11), 55-61.

Figueiredo, S. (2010). Educación musical en la escuela brasileña: Aspectos históricos, legislación educacional y desafíos contemporáneos. Estudios pedagógicos, 64(214), 36-51. doi: https://doi.org/10.4067/ S0716-27902010000200005

Freire, P. (1985). Pedagogía del oprimido. Madrid: Siglo XXI.

Georgii-Hemming, E. y Westvall, M. (2010). Music education - a personal matter? Examining the current discourses of music education in Sweden. British journal of music education, 27(1), 21-33. doi: https://doi.org/10.1017/ S0265051709990179

Giráldez, A. y Palacios, A. (2016). Demandas formativas sobre la educación artística en Iberoamérica. Valencia: Universidad Internacional de Valencia. Recuperado de http://www.viu.es/investigacion/informes/otros/ demandas-formativas-sobre-la-educacion-artistica-en-iberoamerica/

Guerrero, B. (2007). Identidad sociomusical de los jóvenes aymaras: La música sound. Última década, 15(27), 11-25. doi: https://doi.org/10.4067/S0718-22362007000200002

Hatch, M. J. (2013). Organization theory: Modern, symbolic and postmodern perspectives. Oxford: Oxford University Press.

Heimonen, M. y Hebert, D. (2010). Nationalism and music education: A Finnish perspective. En D. Hebert y A. Kerzt-Welzel (Eds.), Patriotism and nationalism in music education (pp. 157-174). Nueva York: Routledge.

Jorquera, M. C. (2010). Modelos didácticos en la enseñanza musical: El caso de la escuela española. Revista musical chilena, 64(214), 52-74. doi: https://doi.org/10.4067/S0716-27902010000200006

Lemberger, M. y Hutchison, B. (2014). Advocating Student-Within-Environment: A humanistic approach for therapists to animate social justice in the schools. Journal of humanistic psychology, 54(1), 28-44. doi: https:// doi.org/10.1177/0022167812469831

Liceras, A. (2014). La educación informal de los medios de comunicación y la protección de los menores de la violencia en televisión: Historia de un fracaso. Profesorado, 18(2), 353-365.

London School of Economics and Political Science. (2012). The impact of three London Conservatoires on the UK and London Economies. Londres: LSE.

Longueira, S. (2013). Los retos educativos en la sociedad del conocimiento. Aproximación a las aportaciones desde el ámbito de la educación musical. Teoría de la educación: Educación y cultura en la sociedad de la información, 14(3), 211-240.

Lorenzo-Quiles, O. y Cárdenas, R. (2010). Antecedentes y actualidad de la música y la educación musical en Colombia. Arte y ciencia, creación y responsabilidad, 2(1), 291-312.

MECD. (2013, 10 de diciembre). Ley Orgánica 8/2013, de 9 de diciembre, para la mejora de la calidad educativa. Boletín Oficial del Estado, 295, 97858-97921.

Morgan, J., MacDonald, R. y Pitts, S. (2015). Caught between a scream and a hug": Women's perspectives on music listening and interaction with teenagers in the family unit. Psychology of music, 43(5), 611-626. doi: https:// doi.org/10.1177/0305735613517411 
OCDE. (2005). Definition and selection of competencies: Theorical and conceptual foundations. Recuperado desde http://www.deseco.admin.ch/bfs/deseco/en/index/03/02.html

OCDE. (2012). Equity and quality in education: Supporting disadvantaged students and schools. OCDE Publishing. doi: https://doi.org/10.1787/9789264130852-en

OCDE. (2016). Education at a glance 2016: OECD indicators. París: OECD Publishing. doi:10.187/eag-2016-en

ONU. (1948, 26 de junio). Carta de las Naciones Unidas y Estatuto de la Corte International de justicia. Lake Success, NY.: Departamento de informaciones Públicas.

OREALC/UNESCO. (2008). Declaración universal de derechos humanos. Santiago: OREALC/UNESCO.

Osses, S., Sánchez, S. y Ibáñez, F. (2006). Investigación cualitativa en educación: Hacia la generación de teoría a través del proceso analítico. Estudios pedagógicos, 32(1), 119-133. doi: https://doi.org/10.4067/ S0718-07052006000100007

Parlamento Europeo y Consejo de la Unión Europea. (2006, 18 de diciembre). Competencias clave para el aprendizaje permanente. Diario Oficial de la Unión Europea, pp. 10-18. Recuperado desde http://eur-lex.europa.eu/legalcontent/ES/TXT/?uri=CELEX:32006H0962

Pitts, S. (2007). Music beyond school: Learning through participation. En L. Bresler (ed.), International handbook of research in Arts Education (pp. 759-772). Dordrecht: Springer. doi: https:// doi.org/10.1007/978-1-4020-3052-9_51

Pitts, S. (2012). Chances and choices: Exploring the impact of music education. Oxford: Oxford University Press. doi: https://doi.org/10.1093/acprof:oso/9780199838752.001.0001

Poblete, C. (2017). Formación docente en música en Chile: Una aproximación histórica desde tres universidades. Educação e Contemporaneidade, 26(48), 97-109. doi: https://doi.org/10.21879/ faeeba2358-0194.2017.v26.n48.p97-109

Samper, A. (2016). La educación musical como derecho humano: Hacia una pedagogía estética, ética y diversa. Cuadernos de Música, Artes Visuales y Artes Escénicas, 11(1), 9-13.

San Martín, D. (2014). Teoría fundamentada y Atlas.ti: Recursos metodológicos para la investigación educativa. Revista electrónica de investigación educativa, 16(1), 104-122.

Schipper, S. (2014). The financial crisis and the hegemony of urban neoliberalism: Lessons from Frankfurt am Main. International journal of urban and regional research, 38(1), 236-255. doi: https:// doi.org/10.1111/1468-2427.12099

Schmidt, P. (2015). Why a social justice vision of Music Education requires a commitment to policy thought. En C. Benedict, P. Schmidt, G. Spruce y P. Woodford (Eds.), Social justice and Music Education (pp. 47-61). Nueva York, NY: Oxford University Press.

Söderman, J. y Sernhede, O. (2016). Hip-hop - what's in it for the academy? Self-understanding, pedagogy and aesthetical learning processes in everyday cultural Praxis. Music education research, 18(2), 142-155. doi: https:// doi.org/10.1080/14613808.2015.1049257

Springer, D. G. (2015). Teaching popular music: Investigating music educators' perceptions and preparation. International journal of music education, 34(4), 403-415. doi: https://doi.org/10.1177/0255761415619068

Tobón, S. (2005). Formación basada en competencias: Pensamiento complejo, diseño curricular y didáctica. Bogotá: Ecoe.

Touriñán, J. y Longueira, S. (2009). Formación de criterio a favor de la música como ámbito de educación. Bordón, 61(1), 43-59.

UNESCO. (1990, marzo). Declaración mundial sobre educación para todos. Conferencia mundial sobre Educación para Todos (pp. 1-11). Jomtien: UNESCO.

UNESCO. (2008). Un enfoque de la educación para todos basado en los derechos humanos. París: UNESCO.

UNESCO. (2010, mayo). La agenda de Seúl: Objetivos para el desarrollo de la educación artística. Resultado de la Segunda Conferencia Mundial sobre la Educación Artística, Seúl, Corea.

UNESCO. (2011). La UNESCO y la educación: "Toda persona tiene derecho a la educación". París: UNESCO. 
Rolando Angel -Alvarado. La Crisis de la educación Musical como consecuencia de la decadenCia DE L...

UNESCO. (2014). Enseñanza y aprendizaje: Lograr la calidad para todos. París: UNESCO.

UNESCO. (2015). Replantear la educación: ¿Hacia un bien común mundial? París: UNESCO.

Vaillant, D. (2013). Formación inicial del profesorado en América Latina: Dilemas centrales y perspectivas. Revista española de educación comparada, 22, 185-206. doi: https://doi.org/10.5944/reec.22.2013.9329

Vierger, M. (2014). Gestión, difusión y enseñanza online en las orquestas sinfónicas actuales. El proyecto enclavemusical/UPNA y la orquesta sinfónica de Navarra Social. En J. Gonzálvez (Coord.), Nuevas tendencias en innovación educativa superior (pp.71-90). Madrid: ACCI.

CC BY-NC-ND 